\title{
Technetium Tc 94m Sestamibi
}

National Cancer Institute

\section{Source}

National Cancer Institute. Technetium Tc 94m Sestamibi. NCI Thesaurus. Code C48423.

A radioconjug ate consisting of sestamibi labeled with the positron-emitting isotope Tc $94 \mathrm{~m}$. Sestamibi is a synthetic molecule of the isonitrile family that diffuses through cell membranes and may preferentially accumulate within mitochondria. Technetium Tc 94m sestamibi may be used in tumor-imaging studies utilizing positron emission tomography (PET). (NCI05) 